PROCEEDINGS OF THE

AMERICAN MATHEMATICAL SOCIETY

Volume 136, Number 7, July 2008, Pages 2597-2607

S 0002-9939(08)09163-6

Article electronically published on March 4, 2008

\title{
PACKING DIMENSION OF THE RANGE OF A LÉVY PROCESS
}

\author{
DAVAR KHOSHNEVISAN AND YIMIN XIAO
}

(Communicated by Richard C. Bradley)

\begin{abstract}
Let $\{X(t)\}_{t \geq 0}$ denote a Lévy process in $\mathbf{R}^{d}$ with exponent $\Psi$. Taylor (1986) proved that the packing dimension of the range $X([0,1])$ is given by the index

$$
\gamma^{\prime}=\sup \left\{\alpha \geq 0: \liminf _{r \rightarrow 0^{+}} \int_{0}^{1} \frac{\mathrm{P}\{|X(t)| \leq r\}}{r^{\alpha}} d t=0\right\} .
$$

We provide an alternative formulation of $\gamma^{\prime}$ in terms of the Lévy exponent $\Psi$. Our formulation, as well as methods, are Fourier-analytic, and rely on the properties of the Cauchy transform. We show, through examples, some applications of our formula.
\end{abstract}

\section{INTRODUCTION}

Let $X:=\{X(t)\}_{t \geq 0}$ denote a $d$-dimensional Lévy process [2], 20] which starts at the origin. Define $\Psi$ to be the Lévy exponent of $X$, normalized so that $\mathrm{E}[\exp (i z \cdot X(t))]=\exp (-t \Psi(z))$ for all $t \geq 0$ and $z \in \mathbf{R}^{d}$, and let $\operatorname{dim}_{\mathrm{P}}$ denote the packing dimension 24, 22]. S. J. Taylor 23, has proved that with probability one, $\operatorname{dim}_{\mathrm{P}} X([0,1])=\gamma^{\prime}$, where $\gamma^{\prime}$ is the index of Hendricks [8]; see $(0.1)$.

Usually, one defines a Lévy process by constructing its Lévy exponent $\Psi$. From this perspective, formula (0.1) is difficult to apply in concrete settings. Primarily this is because the small- $r$ behavior of $\int_{0}^{1} \mathrm{P}\{|X(t)| \leq r\} d t$ is only well-understood when $X$ is a nice Lévy process. For instance, when $X$ is a subordinator $\gamma^{\prime}$ can be shown to be equal to the Blumenthal and Getoor [4] upper index $\beta$ [7, 3]; see also Theorem 3.3 below. When $X$ is a general Lévy process, Pruitt and Taylor [18] find several quantitative relationships between $\gamma^{\prime}$ and other known fractal indices of Lévy processes.

The principal goal of this article is to describe $\gamma^{\prime}=\operatorname{dim}_{\mathrm{P}} X([0,1])$ more explicitly than (0.1), and solely in terms of the Lévy exponent $\Psi$. For all $r>0$ define

$$
W(r):=\int_{\mathbf{R}^{d}} \frac{\kappa(x / r)}{\prod_{j=1}^{d}\left(1+x_{j}^{2}\right)} d x
$$

Received by the editors June 21, 2006, and, in revised form, January 25, 2007, and March 1, 2007 .

2000 Mathematics Subject Classification. Primary 60J30, 60G17, 28A80.

Key words and phrases. Lévy processes, operator stable Lévy processes, packing dimension, Hausdorff dimension.

This research was partially supported by a grant from the National Science Foundation.

(C)2008 American Mathematical Society Reverts to public domain 28 years from publication 
where $\kappa$ is the following well-known function [16, [11:

$$
\kappa(z):=\operatorname{Re}\left(\frac{1}{1+\Psi(z)}\right) \quad \text { for all } z \in \mathbf{R}^{d} .
$$

This function is symmetric (i.e., $\kappa(-z)=\kappa(z)$ for all $z \in \mathbf{R}^{d}$ ) and satisfies the pointwise bounds $0 \leq \kappa \leq 1$, which we use tacitly throughout. The following contains our formula for $\gamma^{\prime}$.

Theorem 1.1. For all d-dimensional Lévy processes $X$,

$$
\operatorname{dim}_{\mathrm{P}} X([0,1])=\sup \left\{\alpha \geq 0: \liminf _{r \rightarrow 0^{+}} \frac{W(r)}{r^{\alpha}}=0\right\}=\limsup _{r \rightarrow 0^{+}} \frac{\log W(r)}{\log r},
$$

almost surely, where $\sup \varnothing:=0$.

Xiao 25, Question 4.16] has asked if we can write $\operatorname{dim}_{\mathrm{P}} X([0,1])$ explicitly in terms of $\Psi$. Theorem 1.1 answers this question in the affirmative.

The following is one of the many consequences of Theorem 1.1

Theorem 1.2. Let $X$ be a d-dimensional Lévy process that has a non-trivial, nondegenerate Gaussian part. That is, $X=G+Y$, where $G$ is a non-degenerate Gaussian Lévy process, and $Y$ is an independent pure-jump Lévy process. Then, $\operatorname{dim}_{\mathrm{P}} X([0,1])=\operatorname{dim}_{\mathrm{P}} G([0,1])$ and $\operatorname{dim}_{\mathrm{H}} X([0,1])=\operatorname{dim}_{\mathrm{H}} G([0,1])$ a.s., where $\operatorname{dim}_{\mathrm{H}}$ denotes the Hausdorff dimension.

We do not know of a direct proof of this result, although it is a very natural statement. However, some care is needed, as the result can fail when $G$ is degenerate (Example 4.1). Our methods will make clear that in general we can say only that $\operatorname{dim} X([0,1]) \geq \operatorname{dim} G([0,1])$ a.s., where "dim" stands for either " $\operatorname{dim}_{\mathrm{P}}$ " or " $\operatorname{dim}_{\mathrm{H}}$."

We also mention the following ready consequence of Theorem 1.1 .

Corollary 1.3. Let $X$ be a Lévy process in $\mathbf{R}^{d}$ and $X^{\prime}(t):=X(t)-X^{\prime \prime}(t)$, where $X^{\prime \prime}$ is an independent copy of $X$. Then, $\operatorname{dim}_{\mathrm{P}} X([0,1]) \geq \operatorname{dim}_{\mathrm{P}} X^{\prime}([0,1])$ a.s.

It has been shown that Corollary 1.3 continues to hold if we replace $\operatorname{dim}_{\mathrm{P}}$ by $\operatorname{dim}_{\mathrm{H}}$ everywhere; see Khoshnevisan, Xiao, and Zhong [13] and/or (1.4) below. Thus we have further confirmation of the somewhat heuristic observation of Kesten 11, p. 7] that the range of $X$ is larger than the range of its symmetrization.

Theorem 1.1 is proved in Section 2 Our proof also yields the following almostsure formula for the Hausdorff dimension of $X([0,1])$ :

$$
\operatorname{dim}_{\mathrm{H}} X([0,1])=\sup \left\{\alpha \geq 0: \limsup _{r \rightarrow 0^{+}} \frac{W(r)}{r^{\alpha}}=0\right\}=\liminf _{r \rightarrow 0^{+}} \frac{\log W(r)}{\log r} ;
$$

see Remark 2.4. Recently, Khoshnevisan, Xiao, and Zhong 13 established an equivalent formulation of this formula. Whereas their derivation is long and complicated, ours is direct and fairly elementary. Section 3 contains non-trivial examples wherein we compute $\operatorname{dim}_{\mathrm{p}} X([0,1])$ for anisotropic Lévy processes $X$. Finally, Theorem 1.2 is proved in Section 4 .

\section{The incomplete Renewal measure}

Define $U$ to be the incomplete renewal measure of $X$. That is, for all Borel sets $A \subset \mathbf{R}^{d}$,

$$
U(A):=\int_{0}^{1} \mathrm{P}\{X(t) \in A\} d t
$$


We may deduce from (0.1) that

$$
\operatorname{dim}_{\mathrm{P}} X([0,1])=\limsup _{r \rightarrow 0^{+}} \frac{\log U(B(0, r))}{\log r},
$$

where $B(a, r):=\left\{z \in \mathbf{R}^{d}:|z-a| \leq r\right\}$ for all $a \in \mathbf{R}^{d}$ and $r \geq 0$ so that $\int_{0}^{1} \mathrm{P}\{|X(t)| \leq r\} d t=U(B(0, r))$, and where $|y|:=\max _{1 \leq j \leq d}\left|y_{j}\right|$ is the box norm of $y \in \mathbf{R}^{d}$.

Let $\zeta$ denote an independent, mean-one exponential random variable. The killed occupation measure of $B(0, r)$ can then be defined by

$$
T(r):=\int_{0}^{\zeta} \mathbf{1}_{B(0, r)}(X(t)) d t \quad \forall r>0,
$$

where $\mathbf{1}_{A}$ denotes the indicator function of $A$.

Proposition 2.1. For all $r>0$,

$$
\frac{e}{4^{d}(e-1)} \mathrm{E}[T(r)] \leq U(B(0, r)) \leq e \mathrm{E}[T(r)] .
$$

In order to prove this we first recall the notion of weak unimodality [12.

Definition 2.2. A Borel measure $\mu$ on $\mathbf{R}^{d}$ is c-weakly unimodal if $c>0$ is a constant that satisfies $\sup _{a \in \mathbf{R}^{d}} \mu(B(a, r)) \leq c \mu(B(0, r))$ for all $r>0$.

The following is a variant of Lemma 4.1 of Khoshnevisan and Xiao 12 .

Lemma 2.3. $U$ is $4^{d}$-weakly unimodal.

Proof. Let us fix $a \in \mathbf{R}^{d}$ and $r>0$, and define $\sigma:=\inf \{s>0:|X(s)-a| \leq r\}$, where $\inf \varnothing:=\infty$. Clearly, $\sigma$ is a stopping time, and

$$
U(B(a, r))=\mathrm{E}\left[\int_{\sigma}^{1} \mathbf{1}_{B(a, r)}(X(s)) d s ; \sigma \leq 1\right] .
$$

Thanks to the triangle inequality, the strong Markov property implies that

$$
\begin{aligned}
U(B(a, r)) & =\mathrm{E}\left[\int_{0}^{(1-\sigma)^{+}} \mathbf{1}_{B(0, r)}(X(u+\sigma)-a) d u\right] \\
& \leq \mathrm{E}\left[\int_{0}^{1} \mathbf{1}_{B(0,2 r)}(X(u+\sigma)-X(\sigma)) d u\right]=U(B(0,2 r)) .
\end{aligned}
$$

Euclidean topology in the box norm dictates that there are points $z_{1}, \ldots, z_{4^{d}} \in$ $B(0,2 r)$ that have the property that $\bigcup_{i=1}^{4^{d}} B\left(z_{i}, r / 2\right)=B(0,2 r)$. According to (2.6), we have the following "volume doubling" property:

$$
U(B(0,2 r)) \leq \sum_{i=1}^{4^{d}} U\left(B\left(z_{i}, r / 2\right)\right) \leq 4^{d} U(B(0, r)) .
$$

The desired result follows from this and (2.6). 
Proof of Proposition 2.1. Note that

$$
U(B(0, r)) \leq e \int_{0}^{1} \mathrm{P}\{|X(t)| \leq r\} e^{-t} d t \leq e \mathrm{E}[T(r)] .
$$

This proves the upper bound in (2.4). To prove the other half we note that

$$
\mathrm{E}[T(r)]=\int_{0}^{\infty} \mathrm{P}\{|X(t)| \leq r\} e^{-t} d t \leq \sum_{j=0}^{\infty} e^{-j} \mathrm{E}[U(B(X(j), r))],
$$

thanks to the Markov property. By Lemma 2.3. $\mathrm{E}[U(B(X(j), r))] \leq 4^{d} U(B(0, r))$ for every $j \geq 0$. The lower bound in (2.4) follows from this and (2.9).

Proof of Theorem 1.1. We derive only the second identity of (1.3); the first is manifestly an equivalent statement.

Let $(\mathscr{F} f)(z):=\int_{\mathbf{R}^{d}} e^{i z \cdot x} f(x) d x$ denote the Fourier transform of $f \in L^{1}\left(\mathbf{R}^{d}\right)$. For all fixed $r>0$ and $x \in \mathbf{R}^{d}$ define

$$
\phi_{r}(x)=\prod_{j=1}^{d} \frac{1-\cos \left(2 r x_{j}\right)}{2 \pi r x_{j}^{2}} .
$$

Then $\phi_{r}(x) \geq 0$, and $\left(\mathscr{F} \phi_{r}\right)(z)=\prod_{j=1}^{d}\left(1-\left|z_{j}\right| /(2 r)\right)^{+}$for all $z \in \mathbf{R}^{d}$ [6, p. 94]. As usual, $a^{+}:=\max (a, 0)$ for all $a \in \mathbf{R}$. Evidently, $\phi_{r} \in L^{1}\left(\mathbf{R}^{d}\right)$, and $0 \leq \mathscr{F} \phi_{r} \leq 1$ pointwise.

Note that $z \in B(0, r)$ implies that $1-(2 r)^{-1}\left|z_{j}\right| \geq \frac{1}{2}$. This implies that $\mathbf{1}_{B(0, r)}(z) \leq 2^{d}\left(\mathscr{F} \phi_{r}\right)(z)$ for all $z \in \mathbf{R}^{d}$. Therefore, by the Fubini-Tonelli theorem,

$$
\mathrm{E}[T(r)] \leq 2^{d} \int_{\mathbf{R}^{d}} \kappa(x) \phi_{r}(x) d x \leq 2^{d} W(r) .
$$

The last inequality follows from the elementary bound

$$
\frac{1-\cos (2 u)}{2 \pi u^{2}} \leq \frac{1}{1+u^{2}} \quad \text { for all } u \in \mathbf{R} .
$$

This can be verified by considering $|u| \leq(\pi-1)^{-1 / 2}$ and $|u|>(\pi-1)^{-1 / 2}$ separately. Thanks to (2.2) and Proposition 2.1,

$$
\operatorname{dim}_{\mathrm{P}} X([0,1]) \geq \limsup _{r \rightarrow 0^{+}} \frac{\log W(r)}{\log r} \quad \text { a.s. }
$$

In order to establish the converse inequality we introduce the process $\{S(t)\}_{t \geq 0}$ defined by $S(t):=\left(S_{1}(t), \ldots, S_{d}(t)\right)$, where $S_{1}, \ldots, S_{d}$ are independent symmetric Cauchy processes in $\mathbf{R}$, all with the same characteristic function $\mathrm{E}\left[e^{i z S_{1}(t)}\right]=e^{-t|z|}$. We assume further that $S$ is independent of $X$. Then for all $\lambda>0$,

$$
\mathrm{E}[\exp \{i X(t) \cdot S(\lambda)\}]=\mathrm{E}\left[\exp \left\{-\lambda \sum_{j=1}^{d}\left|X_{j}(t)\right|\right\}\right] .
$$


On the other hand, the scaling property of $S$ implies

$$
\mathrm{E}\left[e^{i X(t) \cdot S(\lambda)}\right]=\mathrm{E}\left[e^{-t \Psi(S(\lambda))}\right]=\frac{1}{\pi^{d}} \int_{\mathbf{R}^{d}} \frac{e^{-t \Psi(\lambda x)}}{\prod_{j=1}^{d}\left(1+x_{j}^{2}\right)} d x .
$$

For all $r, k>0$ and $x \in \mathbf{R}^{d}$, $\exp \left\{-(k / r) \sum_{j=1}^{d}\left|x_{j}\right|\right\} \leq \mathbf{1}_{B(0, r)}(x)+e^{-k} \mathbf{1}_{B(0, r)^{c}}(x)$. Therefore, $\mathbf{1}_{B(0, r)}(x) \geq \mathrm{E}[\exp \{i x \cdot S(k / r)\}]-e^{-k} \mathbf{1}_{B(0, r)^{c}}(x)$, whence

$$
\mathrm{E}[T(r)] \geq \int_{0}^{\infty} \mathrm{E}[\exp \{i X(t) \cdot S(k / r)\}] e^{-t} d t-e^{-k}(1-\mathrm{E}[T(r)]) .
$$

This and (2.14) together with the fact that the quantity in (2.14) is real imply that

$$
\left(1-e^{-k}\right) \mathrm{E}[T(r)] \geq-e^{-k}+\frac{1}{\pi^{d}} W(r / k) .
$$

Now we choose $k=r^{-\varepsilon}$, for an arbitrary small $\varepsilon>0$, to find that the inequality in (2.13) is an equality. This completes our proof.

Remark 2.4. From the proof of Theorem 1.1 we see that $\mathrm{E}[T(r)]$ and $W(r)$ are roughly comparable; i.e., for all $\varepsilon, r>0$ sufficiently small,

$$
\frac{1}{\pi^{d}} W\left(r^{1+\varepsilon}\right)-\exp \left(-r^{-\varepsilon}\right) \leq \mathrm{E}[T(r)] \leq 2^{d} W(r) .
$$

Thanks to Proposition 2.1 this yields (1.4).

\section{Some EXAMPLES}

We illustrate the utility of Theorem 1.1 by specializing it to a large class of examples.

3.1. Anisotropic examples. It is possible to construct examples of anisotropic Lévy "stable-like" processes whose $\operatorname{dim}_{\mathrm{P}} X([0,1])$ are computable. The following furnishes most of the basic technical background that we shall need.

Theorem 3.1. Let $X$ be a Lévy process in $\mathbf{R}^{d}$ with Lévy exponent $\Psi$. Suppose there exist constants $\beta_{1}, \ldots, \beta_{d}$ such that

$$
2 \geq \beta_{1} \geq \cdots \geq \beta_{d}>0 \text { and } \lim _{\|z\| \rightarrow \infty} \frac{1}{\ln \|z\|} \ln \operatorname{Re}\left(\frac{\sum_{j=1}^{d}\left|z_{j}\right|^{\beta_{j}}}{1+\Psi(z)}\right)=0 .
$$

If $N:=\max \left\{1 \leq j \leq d: \beta_{j}=\beta_{1}\right\}$, then almost surely,

$$
\operatorname{dim}_{\mathrm{P}} X([0,1])= \begin{cases}\beta_{1}, & \text { if } \beta_{1} \leq N, \\ 1+\beta_{2}\left(1-\frac{1}{\beta_{1}}\right), & \text { otherwise }\end{cases}
$$

Proof. Throughout this proof we write $c$ and $C$ for generic constants whose values can change between lines. In order to simplify the exposition somewhat, we note that it is sufficient to prove (3.2) under the following [slightly] stronger form of (3.1):

$$
\frac{c}{1+\sum_{j=1}^{d}\left|z_{j}\right|^{\beta_{j}}} \leq \operatorname{Re}\left(\frac{1}{1+\Psi(z)}\right) \leq \frac{C}{1+\sum_{j=1}^{d}\left|z_{j}\right|^{\beta_{j}}} \quad \text { for all } z \in \mathbf{R}^{d} .
$$


First we consider the case when $\beta_{1} \leq N$. Condition (3.3) implies that if $r \in$ $(0,1)$, then

$$
W(r) \geq c r^{\beta_{1}} \int_{\mathbf{R}^{d}} \frac{d x}{\left(1+\sum_{j=1}^{d}\left|x_{j}\right|^{\beta_{j}}\right) \prod_{j=1}^{d}\left(1+x_{j}^{2}\right)}=C r^{\beta_{1}}
$$

Hence we have $\lim _{r \rightarrow 0} r^{-\alpha} W(r)=\infty$ for all $\alpha>\beta_{1}$. It follows from this and Theorem 1.1 that $\operatorname{dim}_{\mathrm{P}} X([0,1]) \leq \beta_{1}$ a.s.

Recall that $N:=\max \left\{1 \leq j \leq d: \beta_{j}=\beta_{1}\right\}$. From this it follows that

$$
\begin{aligned}
W(r) & \leq c \int_{\mathbf{R}^{N}} \frac{d x}{\left(1+\|x / r\|^{\beta_{1}}\right) \prod_{j=1}^{N}\left(1+x_{j}^{2}\right)} \\
& =c r^{\beta_{1}}\left[\int_{\|x\| \leq 1} \frac{d x}{r^{\beta_{1}}+\|x\|^{\beta_{1}}}+C\right] \leq c r^{\beta_{1}} \log (1 / r) .
\end{aligned}
$$

In the above, $\log (1 / r)$ accounts for the case that $\beta_{1}=N$. The preceding bound implies that $\lim _{r \rightarrow 0} r^{-\alpha} W(r)=0$ for every $\alpha<\beta_{1}$. This leads to the lower bound, $\operatorname{dim}_{\mathrm{P}} X([0,1]) \geq \beta_{1}$ a.s.

Next we consider the case when $\beta_{1}>N$. This implies $N=1$ and $\beta_{1}>\beta_{2}$. In order to prove that $\operatorname{dim}_{\mathrm{P}} X([0,1]) \leq 1+\beta_{2}\left(1-\beta_{1}^{-1}\right)$ a.s. we first derive a lower bound for $W(r)$. We do this by restricting the integral to the domain $D:=\{x \in$ $\mathbf{R}^{d}:\left|x_{j}\right| \leq 1$ for $\left.3 \leq j \leq d\right\}$. More precisely, by (3.3) we have

$$
\begin{aligned}
W(r) & \geq c \int_{D} \frac{d x}{\left(1+\sum_{j=1}^{d}\left|x_{j} / r\right|^{\beta_{j}}\right) \prod_{j=1}^{d}\left(1+x_{j}^{2}\right)} \\
& \geq c \int_{D} \frac{d x}{\left(1+\sum_{j=1}^{d}\left|x_{j} / r\right|^{\beta_{j}}\right) \prod_{j=1}^{2}\left(1+x_{j}^{2}\right)} .
\end{aligned}
$$

Let $\left(x_{3}, \ldots, x_{d}\right) \in[-1,1]^{d-2}$ be fixed and let $A:=1+\sum_{j=3}^{d}\left|x_{j} / r\right|^{\beta_{j}}$. Consider

$$
\begin{aligned}
\mathscr{I} & :=\int_{\mathbf{R}^{2}} \frac{d x_{1} d x_{2}}{\left(A+\left|x_{1} / r\right|^{\beta_{1}}+\left|x_{2} / r\right|^{\beta_{2}}\right) \prod_{j=1}^{2}\left(1+x_{j}^{2}\right)} \\
& \geq r^{\beta_{2}} \int_{r^{1-\left(\beta_{2} / \beta_{1}\right)}}^{\infty} d x_{1} \int_{0}^{\infty} \frac{d x_{2}}{\left(r^{\beta_{2}} A+r^{\beta_{2}-\beta_{1}} x_{1}^{\beta_{1}}+x_{2}^{\beta_{2}}\right) \prod_{j=1}^{2}\left(1+x_{j}^{2}\right)} .
\end{aligned}
$$

Observe that $r^{\beta_{2}-\beta_{1}} x_{1}^{\beta_{1}} \geq 1$ for all $x_{1} \geq r^{1-\left(\beta_{2} / \beta_{1}\right)}$. On the other hand, $r^{\beta_{2}} A \leq d-1$ for all $r \in(0,1)$. It follows from these facts, and a change of variables, that

$$
\begin{aligned}
\mathscr{I} & \geq c r^{\beta_{2}} \int_{r^{1-\left(\beta_{2} / \beta_{1}\right)}}^{\infty} \frac{d x_{1}}{1+x_{1}^{2}} \int_{1}^{\infty} \frac{1}{\left(r^{\beta_{2}-\beta_{1}} x_{1}^{\beta_{1}}+x_{2}^{\beta_{2}}\right) x_{2}^{2}} d x_{2} \\
& \geq c r^{\beta_{2}} \int_{r^{1-\left(\beta_{2} / \beta_{1}\right)}}^{\infty} \frac{d x_{1}}{1+x_{1}^{2}} \cdot \frac{1}{r^{\beta_{2}-\beta_{1}} x_{1}^{\beta_{1}}} \geq c r^{\beta_{1}} \int_{r^{1-\left(\beta_{2} / \beta_{1}\right)}}^{1} \frac{d x_{1}}{x_{1}^{\beta_{1}}} \\
& \geq C r^{1+\beta_{2}\left(1-\beta_{1}^{-1}\right)} .
\end{aligned}
$$

Combine this with (3.6) and (3.7) to deduce that $W(r) \geq c r^{1+\beta_{2}\left(1-\beta_{1}^{-1}\right)}$ for all $r \in(0,1)$, whence $\lim _{r \rightarrow 0^{+}} r^{-\alpha} W(r)=\infty$ for all $\alpha>1+\beta_{2}\left(1-\beta_{1}^{-1}\right)$. This implies that $\operatorname{dim}_{\mathrm{P}} X([0,1]) \leq 1+\beta_{2}\left(1-\beta_{1}^{-1}\right)$ a.s. 
Now we derive the lower bound for $\operatorname{dim}_{\mathrm{P}} X([0,1])$. It follows from (3.3) that

$$
\begin{aligned}
W(r) & \leq c \int_{\mathbf{R}^{2}} \frac{d x_{1} d x_{2}}{\left(1+\left|x_{1} / r\right|^{\beta_{1}}+\left|x_{2} / r\right|^{\beta_{2}}\right) \prod_{j=1}^{2}\left(1+x_{j}^{2}\right)} \\
& =4 c r^{\beta_{2}} \int_{0}^{\infty} \frac{d x_{1}}{1+x_{1}^{2}} \int_{0}^{\infty} \frac{d x_{2}}{\left(B+x_{2}^{\beta_{2}}\right)\left(1+x_{2}^{2}\right)},
\end{aligned}
$$

where $B:=r^{\beta_{2}}+r^{\beta_{2}-\beta_{1}} x_{1}^{\beta_{1}}$. It remains to verify that the last expression in (3.9) is at most $c r^{1+\beta_{2}\left(1-\beta_{1}^{-1}\right)} \log (1 / r)$, where $\log (1 / r)$ appears because of the possibility that $\beta_{2}=1$.

By breaking the $d x_{2}$-integral according to whether $\left|x_{2}\right| \leq 1$ or $\left|x_{2}\right|>1$, and after a change of variables, we can verify the following elementary inequalities:

(i) If $B \leq 1$, then $\int_{0}^{\infty}\left(B+x_{2}^{\beta_{2}}\right)^{-1}\left(1+x_{2}^{2}\right)^{-1} d x_{2} \leq c g\left(x_{1}\right)$, where: $g\left(x_{1}\right)=1$ if $\beta_{2}<1, \log \left(B^{-1}\right)$ if $\beta_{2}=1$, and $B^{\left(1 / \beta_{2}\right)-1}$ if $\beta_{2}<1$.

(ii) If $B>1$, then $\int_{0}^{\infty}\left(B+x_{2}^{\beta_{2}}\right)^{-1}\left(1+x_{2}^{2}\right)^{-1} d x_{2} \leq c / B$.

We return to (3.9) and split the $d x_{1}$-integral respectively over the intervals $\left\{x_{1}\right.$ : $B \leq 1\}$ and $\left\{x_{1}: B>1\right\}$. It follows from (3.9), (i) and (ii), and a direct computation, that $W(r) \leq c r^{1+\beta_{2}\left(1-\beta_{1}^{-1}\right)} \log (1 / r)$. Hence, Theorem 1.1 implies that $\operatorname{dim}_{\mathrm{P}} X([0,1]) \geq 1+\beta_{2}\left(1-\beta_{1}^{-1}\right)$ a.s. This finishes the proof Theorem 3.1 .

In the following, we apply Theorem 3.1 to operator-stable Lévy processes in $\mathbf{R}^{d}$ with $d \geq 2$. Let us first recall from [21] that a non-degenerate distribution $\mu$ on $\mathbf{R}^{d}$ is called operator-stable if there exist sequences of independent identically distributed random vectors $\left\{X_{n}\right\}$ in $\mathbf{R}^{d}$, non-singular linear operators $\left\{A_{n}\right\}$, and vectors $\left\{a_{n}\right\}$ in $\mathbf{R}^{d}$ such that $\left\{A_{n} \sum_{k=1}^{n} X_{k}-a_{n}\right\}$ converges in law to $\mu$. A distribution $\mu$ on $\mathbf{R}^{d}$ is called full if it is not supported on any $(d-1)$-dimensional hyper-plane. Sharpe 21 proves that a full distribution $\mu$ in $\mathbf{R}^{d}$ is operator-stable if and only if there exists a non-singular linear operator $B$ on $\mathbf{R}^{d}$ such that $\mu^{t}=t^{B} \mu * \delta(b(t))$ for all $t>0$ and some $b(t) \in \mathbf{R}^{d}$. Here, $\mu^{t}$ denotes the $t$-fold convolution power of $\mu$, and $t^{B} \mu(d x):=\mu\left(t^{-B} d x\right)$ is the image measure of $\mu$ under the action of the linear operator $t^{B}:=\sum_{n=0}^{\infty}(\log t)^{n} B^{n} / n$ !. In the above, $B$ and $\{b(t), t>0\}$ are called a stability exponent and the family of shifts of $\mu$, respectively. The set of all possible exponents of an operator stable law is characterized by Holmes et al. 9]; see also 14, Theorem 7.2.11]. By analogy with the one-dimensional case, an operator-stable distribution $\mu$ satisfying $\mu^{t}=t^{B} \mu$ will be called strictly operator-stable; see [21, p. $64]$.

A stochastic process $Y:=\{Y(t)\}_{t \in \mathbf{R}_{+}}$with values in $\mathbf{R}^{d}$ is said to be operator self-similar if there exists a linear operator $B$ on $\mathbf{R}^{d}$ such that for every $c>0$, $\{Y(c t)\}_{t \geq 0} \stackrel{d}{=}\left\{c^{B} Y(t)\right\}_{t \geq 0}$, where $\stackrel{\text { d }}{=}$ " denotes the equality of finite-dimensional distributions. The linear operator $B$ is called a self-similarity exponent of $Y$. Let $X=\{X(t)\}_{t>0}$ be a Lévy process in $\mathbf{R}^{d}$ starting from 0 such that the distribution of $X(t)$ is full for every $t>0$. Hudson and Mason [10, Theorem 7] proved that $X$ is operator self-similar if and only if the distribution of $X(1), \nu:=\mathrm{P} \circ(X(1))^{-1}$, is strictly operator stable. In this case, every stability exponent $B$ of $\nu$ is also a self-similarity exponent of $X$. Hence, from now on we will call a Lévy process $X$ in $\mathbf{R}^{d}$ operator-stable if the distribution of $X(1)$ is full and strictly operator-stable, and we will refer to $B$ simply as an exponent of $X$. 
Operator-stable Lévy processes are scaling limits of $d$-dimensional random walks that are normalized by linear operators [14, Chapter 11]. All $d$-dimensional strictly stable Lévy processes of index $\alpha$ are operator-stable with exponent $B:=\alpha^{-1} I$, where $I$ denotes the $(d \times d)$ identity matrix.

More generally, let $X_{1}, \ldots, X_{d}$ be independent stable Lévy processes in $\mathbf{R}$ with respective indices $\alpha_{1}, \ldots, \alpha_{d} \in(0,2]$. Define $X(t):=\left(X_{1}(t), \ldots, X_{d}(t)\right)$. One can then verify that $X$ is an operator-stable Lévy process whose exponent $B$ is the $(d \times d)$ diagonal matrix $\operatorname{diag}\left(\alpha_{1}^{-1}, \alpha_{2}^{-1}, \ldots, \alpha_{d}^{-1}\right)$. These processes were first introduced by Pruitt and Taylor [19] under the title of Lévy processes with stable components. These processes have been used to construct various counterexamples 8.

Let $X$ be an operator-stable Lévy process in $\mathbf{R}^{d}$ with exponent $B$. Factor the minimal polynomial of $B$ into $q_{1}(x), \cdots, q_{p}(x)$ where all roots of $q_{i}(x)$ have real part $a_{i}$, and $a_{i}<a_{j}$ for $i<j$. Define $\alpha_{i}:=a_{i}^{-1}$, so that $\alpha_{1}>\cdots>\alpha_{p}$, and note that $0<\alpha_{i} \leq 2$ [14, Theorem 7.2.1]. Define $V_{i}:=\operatorname{Ker}\left(q_{i}(B)\right)$ and $d_{i}:=\operatorname{dim}\left(V_{i}\right)$. Then $d_{1}+\cdots+d_{p}=d$, and $V_{1} \oplus \cdots \oplus V_{p}$ is a direct-sum decomposition of $\mathbf{R}^{d}$ into $B$-invariant subspaces. We may write $B$ as $B=B_{1} \oplus \cdots \oplus B_{p}$, where $B_{i}: V_{i} \rightarrow V_{i}$ and every eigenvalue $\lambda$ of $B_{i}$ has the property that $\operatorname{Re} \lambda=a_{i}$. We can apply Theorem 3.1 for operator stable Lévy processes to obtain a wholly different proof of the following theorem of Meerschaert and Xiao [15, Theorem 3.2].

Theorem 3.2 (Meerschaert and Xiao 15]). Let X be an operator-stable Lévy process in $\mathbf{R}^{d}$. Then almost surely,

$$
\operatorname{dim}_{\mathrm{P}} X([0,1])= \begin{cases}\alpha_{1} & \text { if } \alpha_{1} \leq d_{1}, \\ 1+\alpha_{2}\left(1-\frac{1}{\alpha_{1}}\right) & \text { otherwise }\end{cases}
$$

Proof. Define $\beta_{j}:=\alpha_{\ell}$ where $\ell$ is determined by $\sum_{i=0}^{\ell-1} d_{i}<j \leq \sum_{i=\ell-1}^{\ell} d_{i}$, and $d_{0}:=0$. Because Meerschaert and Xiao [15] have established (3.1), Theorem 3.1 implies (3.10) with $N:=d_{1}$.

3.2. Subordinators. Let us consider the special case that $X$ is a [non-negative] subordinator. We conclude this section by showing that our Theorem 1.1 includes the well known formula for $\operatorname{dim}_{\mathrm{P}} X([0,1])$; see 7 and [3, $\left.\$ 5.1 .2\right]$. Let $\Phi$ denote the Laplace exponent of $X$, normalized so that $\mathrm{E}[\exp (-\lambda X(t))]=\exp (-t \Phi(\lambda))$ for all $\lambda, t \geq 0$. The following is an immediate consequence of Theorem 1.1

Theorem 3.3 (Fristedt and Taylor [7]; Bertoin [3]). With probability one,

$$
\operatorname{dim}_{\mathrm{P}} X([0,1])=\limsup _{\lambda \rightarrow \infty} \frac{\log \Phi(\lambda)}{\log \lambda} .
$$

Proof. Let $S=\{S(t)\}_{t \geq 0}$ denote an independent Cauchy process in $\mathbf{R}$ such that $\mathrm{E}[\exp (i \xi S(t))]=\exp (-t|\xi|)$ for all $t \geq 0$ and $\xi \in \mathbf{R}$. Then,

$$
e^{-t \Phi(\lambda)}=\mathrm{E}\left[e^{-\lambda X(t)}\right]=\mathrm{E}\left[e^{i X(t) S(\lambda)}\right]=\frac{1}{\pi} \int_{-\infty}^{\infty} \frac{e^{-t \Psi(\lambda z)}}{1+z^{2}} d z .
$$

Multiply both sides by $e^{-t}$ and integrate $[d t]$ to find that

$$
\frac{1}{1+\Phi(\lambda)}=\frac{1}{\pi} W(1 / \lambda)
$$

A direct appeal to Theorem 1.1 finishes the proof. 


\section{Proof of Theorem 1.2}

Throughout, $\|x\|:=\left(x_{1}^{2}+\cdots+x_{d}^{2}\right)^{1 / 2}$ for all $x \in \mathbf{R}^{d}$. This is the usual $\ell^{2}$-norm on $\mathbf{R}^{d}$, and should not be confused with the $\ell^{\infty}$-norm $|x|=\max _{1 \leq j \leq d}\left|x_{j}\right|$ that we have used so far.

Because of the Lévy-Khintchine formula [2, we can write $X$ as $X=G+Y$, where $G$ is a Gaussian Lévy process and $Y$ is an independent pure-jump Lévy process. Thanks to the centered-ball inequality of Anderson [1, $a \mapsto \mathrm{P}\{|G(t)-a| \leq r\}$ is maximized at the origin. Apply this, conditionally on $Y$, to find that

$$
\mathrm{P}\{|X(t)| \leq r\} \leq \mathrm{P}\{|G(t)| \leq r\} \quad \text { for all } t, r>0 .
$$

It follows from $(0.1)$ and (2.2) that $\operatorname{dim}_{\mathrm{P}} G([0,1]) \leq \operatorname{dim}_{\mathrm{P}} X([0,1])$ a.s. The analogous bound for $\operatorname{dim}_{\mathrm{H}}$ follows from this and the formula of Pruitt [17].

In order to prove the converse bound we appeal to Theorem 1.1. Recall that

$$
\Psi_{X}(z)=O\left(\|z\|^{2}\right) \quad \text { as }\|z\| \rightarrow \infty
$$

Bochner [5, eq. (3.4.14), p. 67]. The subscript $X$ refers to the process $X$, the subscript $G$ to the process $G$, etc. Therefore, there exists a constant $C$ such that

$$
\kappa_{X}(z) \geq \frac{C}{1+\|z\|^{2}} \quad \text { for all } z \in \mathbf{R}^{d} .
$$

The non-degeneracy of $G$ implies that $\|z\|^{-2} \Psi_{G}(z)$ is bounded below uniformly for all $z \in \mathbf{R}^{d}$. Because $\operatorname{Re} \Psi_{Y}(z) \geq 0$, it follows that there exists a constant $c$ such that $\kappa_{X}(z) \geq c \kappa_{Y}(z)$ for all $z \in \mathbf{R}^{d}$. Therefore, $W_{X}(r) \geq c W_{G}(r)$, and Theorem 1.1 shows that $\operatorname{dim}_{\mathrm{P}} X([0,1]) \leq \operatorname{dim}_{\mathrm{P}} G([0,1])$ a.s. The analogous bound for $\operatorname{dim}_{\mathrm{H}}$ follows from (1.4), Remark 2.4 and Proposition 2.1. This completes the proof.

We conclude this section by mentioning a simple example wherein Theorem 1.2 fails because $G$ is degenerate.

Example 4.1. Let $Y$ be an isotropic stable Lévy process in $\mathbf{R}^{2}$ with index $\alpha \in$ $(1,2]$. Let $G_{1}$ be an independent one-dimensional Brownian motion, and define $G(t):=\left(G_{1}(t), 0\right)$. Then, $X:=G+Y$ has the form of the process in Theorem 1.2, but now $G$ is degenerate. Direct calculations show that $\Psi_{Y}(z)=c\|z\|^{\alpha}$ for some $c>0$, and $\Psi_{G}(z)=c^{\prime} z_{1}^{2}$ for some $c^{\prime}>0$. It follows readily from this discussion that $\Psi_{X}(z)=c\|z\|^{\alpha}+c^{\prime} z_{1}^{2}$, whence it follows that

$$
\frac{A_{1}}{1+\left|z_{1}\right|^{2}+\left|z_{2}\right|^{\alpha}} \leq \kappa_{X}(z) \leq \frac{A_{2}}{1+\left|z_{1}\right|^{2}+\left|z_{2}\right|^{\alpha}} \quad \text { for all } z:=\left(z_{1}, z_{2}\right) \in \mathbf{R}^{2}
$$

where $A_{1}$ and $A_{2}$ are universal constants. Theorem 3.1 implies that with probability one, $\operatorname{dim}_{\mathrm{P}} X([0,1])=1+\alpha\left(1-\frac{1}{2}\right)=1+(\alpha / 2)$. On the other hand, according to Theorem 1.1] with probability one, $\operatorname{dim}_{\mathrm{P}} Y([0,1])=\alpha$, whereas $\operatorname{dim}_{\mathrm{P}} G([0,1])=1$. Therefore, if $\alpha \in(1,2)$, then $\operatorname{dim}_{\mathrm{P}} X([0,1])$ is almost surely strictly greater than both $\operatorname{dim}_{\mathrm{P}} Y([0,1])$ and $\operatorname{dim}_{\mathrm{P}} G([0,1])$.

Despite the preceding, it is not always necessary that $G$ is non-degenerate, viz.,

Example 4.2. Let $Y:=\{Y(t)\}_{t \in \mathbf{R}_{+}}$be a Lévy process in $\mathbf{R}^{d}$ with characteristic exponent $\Psi(\xi)=\|\xi\|^{2} L(\xi)$, where $L: \mathbf{R}^{d} \rightarrow \mathbf{C}$ is slowly varying at infinity. Such exponents can be constructed via the Lévy-Khintchine formula. For any Gaussian process $G:=\{G(t)\}_{t \in \mathbf{R}_{+}}$in $\mathbf{R}^{d}$ define $X:=Y+G$. It follows from Theorem 3.1 that $\operatorname{dim}_{\mathrm{P}} X([0,1])=\operatorname{dim}_{\mathrm{P}} Y([0,1])=\min (2, d)$ almost surely. 


\section{ACKNOWLEDGMENT}

We thank the anonymous referee for his/her careful reading and for making suggestions that improved the exposition of this paper.

\section{REFERENCES}

[1] T. W. Anderson, The integral of a symmetric unimodal function over a symmetric convex set and some probability inequalities, Proc. Amer. Math. Soc. 6 (1955), 170-176. MR0069229 $(16: 1005 \mathrm{a})$

[2] Jean Bertoin, Lévy Processes, Cambridge University Press, Cambridge, 1998. MR.1406564 (98e:60117)

[3] Subordinators: Examples and Applications, Lectures on Probability Theory and Statistics (Saint-Flour, 1997), 1999, pp. 1-91. MR1746300 (2002a:60001)

[4] R. M. Blumenthal and R. K. Getoor, Sample functions of stochastic processes with stationary independent increments, J. Math. Mech. 10 (1961), 493-516. MR0123362 (23:A689)

[5] Salomon Bochner, Harmonic Analysis and the Theory of Probability, University of California Press, Berkeley and Los Angeles, 1955. MR0072370 (17:273d)

[6] Richard Durrett, Probability: Theory and Examples, 2nd edition, Duxbury Press, Belmont, CA, 1996. MR1609153 (98m:60001)

[7] B. E. Fristedt and S. James Taylor, The packing measure of a subordinator, Probab. Th. Rel. Fields 92 (1992), 493-510. MR1169016 (93e:60150)

[8] W. J. Hendricks, A uniform lower bound for Hausdorff dimension for transient symmetric Lévy processes, Ann. Probab. 11 (3) (1983), 589-592. MR704545 (85a:60043)

[9] J. P. Holmes, W. N. Hudson, and J. David Mason, Operator stable laws: Multiple exponents and elliptical symmetry, Ann. Probab. 10 (1982), 602-612. MR659531(83i:60012)

[10] W. N. Hudson and J. David Mason, Operator-self-similar processes in a finite-dimensional space, Trans. Amer. Math. Soc. 273 (1982), 281-297. MR664042 (83h:60051)

[11] Harry Kesten, Hitting probabilities of single points for processes with stationary independent increments, Memoirs of the American Mathematical Society 93, American Mathematical Society, Providence, RI, 1969. MR0272059 (42:6940)

[12] Davar Khoshnevisan and Yimin Xiao, Weak unimodality of finite measures, and an application to potential theory of additive Lévy processes, Proc. Amer. Math. Soc. 131 (8) (2003), 2611-2616 (electronic). MR.1974662 (2004h:60016)

[13] Davar Khoshnevisan, Yimin Xiao, and Yuquan Zhong, Measuring the range of an additive Lévy process, Ann. Probab. 31 (2) (2003), 1097-1141. MR.1964960 (2004c:60155)

[14] Mark M. Meerschaert and H.-P. Scheffler, Limit Distributions for Sums of Independent Random Vectors, Wiley \& Sons, New York, 2001. MR1840531 (2002i:60047)

[15] Mark M. Meerschaert and Yimin Xiao, Dimension results for sample paths of operator stable Lévy processes, Stoch. Proc. Appl. 115 (2005), 55-75. MR2105369 (2005k:60238)

[16] Steven Orey, Polar sets for processes with stationary independent increments, Markov Processes and Potential Theory (Proc. Sympos. Math. Res. Center, Madison, Wis., 1967), Wiley, New York, 1967, pp. 117-126. MR0229305 (37:4879)

[17] William E. Pruitt, The Hausdorff dimension of the range of a process with stationary independent increments, J. Math. Mech. 19 (1969), 371-378. MR0247673 (40:936)

[18] William E. Pruitt and S. James Taylor, Packing and covering indices for a general Lévy process, Ann. Probab. 24 (2) (1996), 971-986. MR1404539 (97g:60096)

[19] _ Sample path properties of processes with stable components, Z. Wahrsch. Verw. Gebiete 12 (1969), 267-289. MR0258126 (41:2773)

[20] Ken-iti Sato, Lévy Processes and Infinitely Divisible Distributions, Cambridge University Press, Cambridge, 1999. Translated from the 1990 Japanese original; revised by the author. MR:1739520 (2003b:60064)

[21] Michael Sharpe, Operator-stable probability distributions on vector groups, Trans. Amer. Math. Soc. 136 (1969), 51-65. MR0238365 (38:6641)

[22] Dennis Sullivan, Entropy, Hausdorff measures old and new, and limit sets of geometrically finite Kleinian groups, Acta Math. 153 (3-4) (1984), 259-277. MR766265 (86c:58093) 
[23] S. James Taylor, The use of packing measure in the analysis of random sets, Stochastic Processes and Their Applications (Nagoya, 1985), Springer, Berlin, 1986, pp. 214-222. MR 872112 (88i:60078)

[24] Claude Tricot Jr., Two definitions of fractional dimension, Math. Proc. Cambridge Philos. Soc. 91 (1) (1982), 57-74. MR633256 (84d:28013)

[25] Yimin Xiao, Random fractals and Markov processes, In: Fractal Geometry and Applications (Michel L. Lapidus and Machiel van Frankenhuijsen, editors), American Mathematical Society, Providence, RI, 2004, pp. 261-338. MR2112126 (2006a:60065)

Department of Mathematics, The University of Utah, 155 S. 1400 East, Salt Lake City, Utah 84112-0090

E-mail address: davar@math.utah.edu

URL: http://www.math.utah.edu/ davar

Department of Statistics and Probability, A-413 Wells Hall, Michigan State UniVersity, East Lansing, Michigan 48824

E-mail address: xiao@stt.msu.edu

$U R L:$ http://www.stt.msu.edu/ xiaoyimi 